\title{
Working Title: Hydrogen Sulfide - Biochemical and Biological Considerations
}

\section{Bogdan Calenic ${ }^{1,2^{*}}$ and Anton Amann ${ }^{3,4^{*}}$}

${ }^{1}$ Department of Biochemistry, Faculty of Dental Medicine, University of Medicine and Pharmacy, Carol Davila, Blvd EroiiSanitari no 8, Bucharest, Romania

${ }^{2}$ Victor Babes, National Institute of Pathology, Biochemistry-Proteomics Department, no 99-101 Splaiul Independentei, 050096, Sector 5 Bucharest, Romania

${ }^{3}$ Breath Research Institute, Leopold-Franzens University of Innsbruck, Rathausplatz 4, A-6850 Dornbirn, Austria

${ }^{4}$ Univ.-Clinic for Anesthesia, Medical University Innsbruck, Anichstrasse 35, A-6020 Innsbruck, Austria

"Corresponding author: Calenic C, Department of Biochemistry, Faculty of Dental Medicine, University of Medicine and Pharmacy Tel: 43-512-504-24636; E-mail: bcalenic@yahoo.co.uk, Amann A, Breath Research Institute, Leopold-Franzens University of Innsbruck, Dornbirn, Austria, Tel: 43-512-504-24636; Fax: 43-512-504-672463; E-mail: anton.amann@i-med.ac.at

Rec date: October 14, 2014, Acc date: December 26, 2014, Pub date: December 29, 2014

Copyright: (C) 2014 Calenic B and Amann A, This is an open-access article distributed under the terms of the Creative Commons Attribution License, which permits unrestricted use, distribution, and reproduction in any medium, provided the original author and source are credited

\begin{abstract}
In the past years biomedical research has recognized hydrogen sulfide $\left(\mathrm{H}_{2} \mathrm{~S}\right)$ not only as an environmental pollutant but also, along with nitric oxide and carbon monoxide, as an important biological gasotransmiter with paramount roles in health and disease. Current research is focused on several aspects of $\mathrm{H}_{2} \mathrm{~S}$ biology such as the biochemical pathways that generate the compound, functions in human pathology or drug synthesis that block or stimulate its biosynthesis. The present editorial addresses the knowledge we have to date on $\mathrm{H}_{2} \mathrm{~S}$ production and its biological roles in the general human environment with a special focus on the oral cavity.
\end{abstract}

Keywords: Hydrogen sulfide; Superoxide dismutase; 3mercaptopyruvate sulfurtransferase; Cystathionine b-synthase; Cystathionine g-lyase

\section{$\mathrm{H}_{2} \mathrm{~S}$ - Generalities}

In the past years biomedical research has recognized hydrogen sulfide $\left(\mathrm{H}_{2} \mathrm{~S}\right)$ not only as an environmental pollutant but also, along with nitric oxide and carbon monoxide, as an important biological gasotransmiter with paramount roles in health and disease. Current research focuses on several aspects of $\mathrm{H}_{2} \mathrm{~S}$ biology such as the biochemical pathways that generate the compound, its functions in human pathology or drug synthesis that block or stimulate its biosynthesis. The present editorial addresses the knowledge we have to date on $\mathrm{H}_{2} \mathrm{~S}$ production and its biological roles in the general human environment with a special focus on the oral cavity.

\section{H2S - Production}

In mammalian organisms, including the human body, enzymatic $\mathrm{H}_{2} \mathrm{~S}$ synthesis is generally connected to three endogenous enzymes: 3mercaptopyruvate sulfurtransferase (3MST), cystathionine b-synthase (CBS) and cystathionine g-lyase (CSE) all three taking part in the cysteine synthesis pathway [1] (Figure 1). It is important to note that each of the three enzymes are responsible for $\mathrm{H}_{2} \mathrm{~S}$ homeostasis and regulates $\mathrm{H}_{2} \mathrm{~S}$ levels found in the bloodstream. Each of the enumerated enzymes is found at specific sites in the organism. Thus 3MST has a mitochondrial location and is usually found in the brain and blood vessels. The enzymes participates in a series of chemical reactions that starts with cysteine metabolism transformed to 3-mercaptopyruvate by cysteine aminotransferase. Further 3-mercaptopyruvate is reacted to pyruvate and finally to $\mathrm{H}_{2} \mathrm{~S}$ by 3 MST. CBS is found mostly in hepatic, cerebral tissues and the nervous system and produces $\mathrm{H}_{2} \mathrm{~S}$ as an end product of a reaction involving cystathionine generation starting from cysteine and serine. CSE resides in blood vessels and hepatic cells and produces $\mathrm{H}_{2} \mathrm{~S}$ trough a reaction that starts with cysteine and generates pyruvate and ammonia. $\mathrm{H}_{2} \mathrm{~S}$ catabolism involves several oxidative steps that converts the gas to persulfide, thiosulfate and sulfate with an oxidation rate this is organ specific. $\mathrm{H}_{2} \mathrm{~S}$ oxidation was shown to occur in virtually all cell types and tissues of the human body including colon, kidney, liver, brain or lung cells [2].

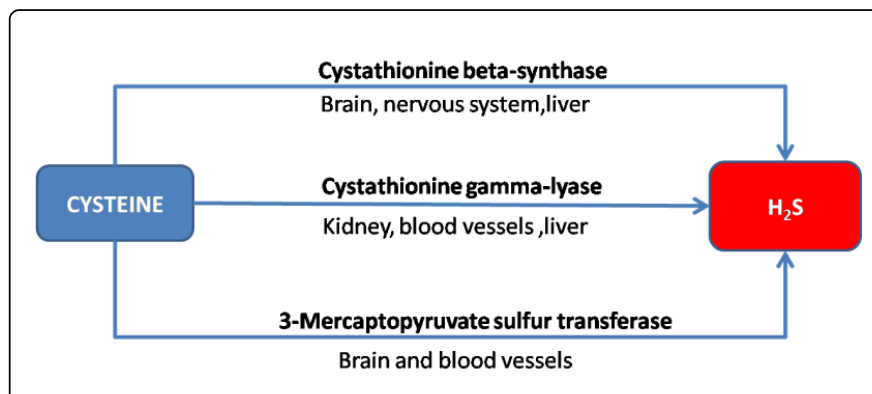

Figure 1: Hydrogen sulfide production - Cysteine biosynthesis pathway is the main pathway responsible for $\mathrm{H}_{2} \mathrm{~S}$ production in mammalian organisms usually with the help of three enzymes.

\section{$\mathrm{H}_{2} \mathrm{~s}$ - Biological Roles}

$\mathrm{H}_{2} \mathrm{~S}$ is a gaseous mediator and with multiple roles depending on the tissue or organ. Thus $\mathrm{H}_{2} \mathrm{~S}$ is involved in blood vessels dilatation, inflammation, cardiac reaction to ischemic injuries. In the human body increased concentrations of $\mathrm{H}_{2} \mathrm{~S}$ are associated with respiratory affections such as chronic bronchitis, emphysema, pneumonia or diseases related to the cardiovascular system such as hypertension [3]. Other volatile sulfur compounds related to $\mathrm{H}_{2} \mathrm{~S}$ (i.e. dimethyl sulfide) were shown to be significantly elevated in patients with cerebrovascular pathology, for example subarachnoid or intracerebral hemorrhages as well as increased cholesterol level, asthma or hepatic affections such as cirrhosis [4](Table 1). 
Page 2 of 3

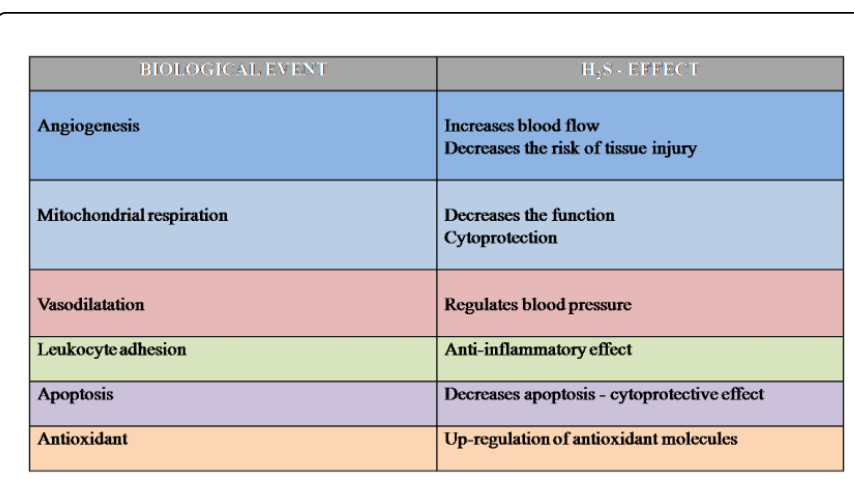

Table 1: Hydrogen sulfide - systemic effects. Adapted after [3].

In the oral environment among other volatile sulfur compounds, $\mathrm{H}_{2} \mathrm{~S}$ is an important compound in the development of physiological and pathological halitosis [5]. Together with periodontal disorders and dental caries, halitosis or oral malodor represents one of the main causes for which patients visit dental practices. Halitosis is the general term used to describe offensive smells detected in human breath. $\mathrm{H}_{2} \mathrm{~S}$ production is mainly caused by metabolic products of oral bacteria that degrade substrates such as cysteine, arginine or tryptophan. Aside its esthetics roles (i.e. involvement in oral malodor) several studies have also focused on the $\mathrm{H}_{2} \mathrm{~S}$ toxicity for the oral environment (Tables 2 and 3 ).

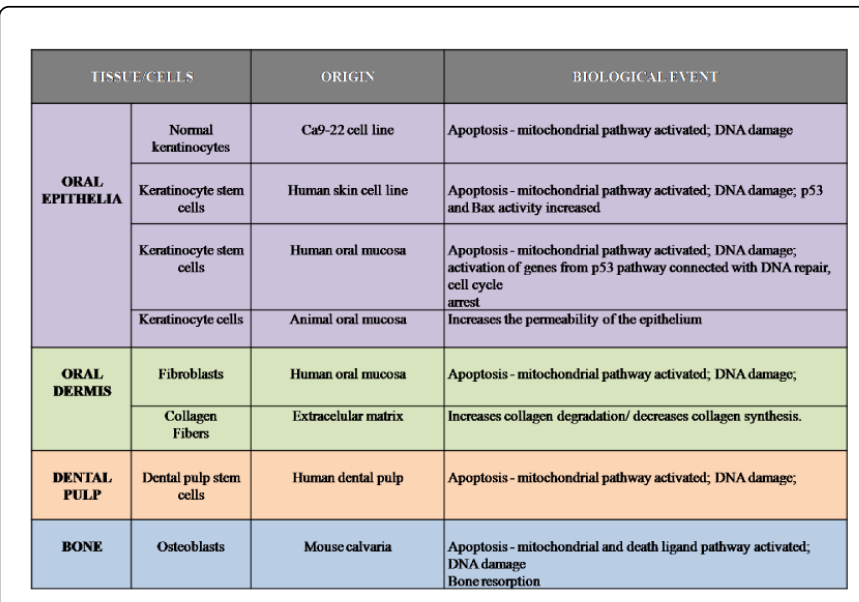

Table 2: Biological effects of high physiological concentrations of $\mathrm{H} 2 \mathrm{~S}$ on different oral cell types (50ng/ml H2S) [7].

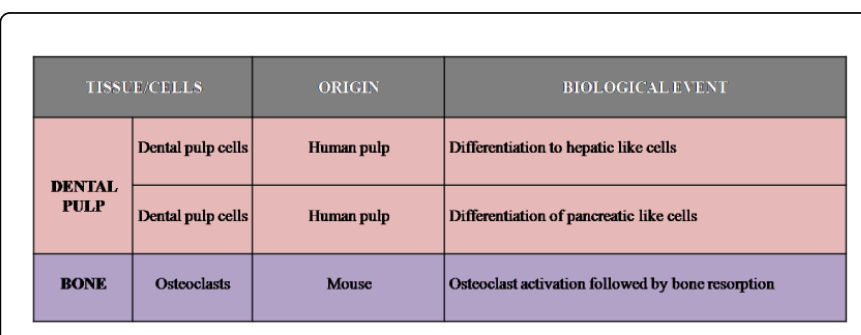

Table 3: Biological effects of low physiological concentrations of $\mathrm{H} 2 \mathrm{~S}$ on different oral cell types (1ng/ml H2S) [7].
Thus reports show that $\mathrm{H}_{2} \mathrm{~S}$ is directly linked to the initiation and development of periodontal diseases: the compound inhibits the proliferation process of oral keratinocyte cells [6], decreases protein synthesis in oral fibroblasts, and inhibits collagen synthesis or basal membrane synthesis [7]. There seem to be a direct relation between the type of the biological effect induced by $\mathrm{H}_{2} \mathrm{~S}$ and the $\mathrm{H}_{2} \mathrm{~S}$ levels. Low physiological concentrations of $\mathrm{H}_{2} \mathrm{~S}$ have been shown to induce dental pulp cells differentiation towards hepatic or pancreatic cells; mouse osteoclasts cells have been shown to switch from a passive state to an active one that induces bone resorption [8]. On the other hand high physiological concentrations of $\mathrm{H}_{2} \mathrm{~S}$ are demonstrated to induce programmed cell death through different molecular pathways in a number of cell types. These concentrations induced apoptosis through inhibition of superoxide dismutase (SOD) in human gingival fibroblasts. This enzyme is paramount in eliminating reactive oxygen species and leads to damage to DNA structure. The same process was observed in normal keratinocytes [9] and keratinocytes stem cells. DNA damage is shown to activate several molecular pathways such as p53 pathway that can decide cellular fate through activation of DNA repair molecules, cell cycle arrest or apoptosis. Volatile sulfur compounds and especially $\mathrm{H}_{2} \mathrm{~S}$ were shown to induce the apoptotic process in several cell types belonging to oral structures. Generally apoptosis follows well established pathways such as: intrinsic mitochondrial pathway where the inner mitochondrial membrane is depolarized followed by cytochrome $\mathrm{C}$ release into cytosol, assembly of the apoptosome that leads to activation of initiator caspase 9 ultimately followed by executioner caspase 3 activation [10]. This pathway was activated in most studied cell types: oral fibroblasts oral keratinocytes, oral keratinocyte stem cells, general keratinocyte stem cells. The extrinsic pathway, or the ligand-activated pathway was shown to be responsible for apoptosis induction only in cells isolated from the alveolar bone, osteoblast cells. At the same time after $\mathrm{H}_{2} \mathrm{~S}$ exposure human oral keratinocyte stem cells expressed key p53-related molecules associated with cell death, DNA repair and cell cycle control.

\section{Future Considerations}

To date there is no general consensus regarding $\mathrm{H}_{2} \mathrm{~S}$ biochemistry and its functions in cell biology (i.e. its pro- or anti-inflammatory effects). In this respect the field can be further expanded together with the development of tools that could correctly identify and quantify $\mathrm{H}_{2} \mathrm{~S}$ synthesis and catabolism in organs and tissues [2]. Another important issue would be the elucidation of endo- or exogenous signals that initiate $\mathrm{H}_{2} \mathrm{~S}$ production together with a better understanding of the chemical pathways responsible for its removal. Overall a more clear understanding of the biochemistry of $\mathrm{H}_{2} \mathrm{~S}$ in relation to its biological roles is greatly needed.

\section{Acknowledgement}

B.C. aknowledges that this paper is partly supported by the Sectorial Operational Programme Human Resources Development (SOPHRD), financed by the European Social Fund and the Romanian Government under the contract number POSDRU 141531. B.C. would also like to thank for the Young Scientist Grant 2011-2013 received from University of Medicine and Pharmacy Carol Davila, Bucharest, Romania. A.A and B.C would also like to acknowledge the support offered by ERASMUS Programme during 2012-2014. 
Citation: $\quad$ Calenic et al. (2015) Working Title: Hydrogen Sulfide - Biochemical and Biological Considerations. J Environ Anal Chem 2: 124. doi: $10.4172 / 2380-2391.1000124$

Page 3 of 3

\section{References}

1. Predmore BL, Lefer DJ, Gojon G (2012) Hydrogen sulfide in biochemistry and medicine. Antioxidants \& redox signaling 17: 119-140.

2. Nagy P, Pálinkás Z, Nagy A, Budai B, Tóth I, et al. (2014) Chemical aspects of hydrogen sulfide measurements in physiological samples. Biochimica et Biophysica Acta (BBA)-General Subjects 1840: 876-891.

3. Calvert JW, Coetzee WA, Lefer DJ (2010) Novel insights into hydrogen sulfide-mediated cytoprotection. Antioxidants \& redox signaling 12: 1203-1217.

4. Li L, Rose P, Moore PK (2011) Hydrogen sulfide and cell signaling. Annual review of pharmacology and toxicology 51: 169-187.

5. Yaegaki K, Brunette DM, Tangerman A (2012) Standardization of clinical protocols in oral malodor research. J. Breath Res 6: 017101.

6. Calenic B, Yaegaki K, Kozhuharova A, Imai T (2010) Oral malodorous compound causes oxidative stress and p53-mediated programmed cell death in keratinocyte stem cells. Journal of periodontology 81: 1317-1323.

7. Calenic B, Amann A (2014) Detection of volatile malodorous compounds in breath: current analytical techniques and implications in human disease. Bioanalysis 6: 357-376.

8. Aoyama I, Calenic B, Imai T, Ii H, Yaegaki K (2012) Oral malodorous compound causes caspase- 8 and -9 mediated programmed cell death in osteoblasts. Journal of periodontal research 47: 365-373.

9. Murata T, Yaegaki K, Qian W (2008) Hydrogen sulfide induces apoptosis in epithelial cells derived from human gingiva. Journal of breath research 2: 017007.

10. Inoue S, Browne G, Melino G, Cohen GM (2009) Ordering of caspases in cells undergoing apoptosis by the intrinsic pathway. Cell death and differentiation 16: 1053-1061. 